\title{
L'économie sociale et solidaire face aux nouvelles attentes de la société
}

The Social and Solidarity Economy and Society's New Expectations

\section{Jean-François Draperi}

Numéro 281, juillet 2001

URI : https://id.erudit.org/iderudit/1024017ar

DOI : https://doi.org/10.7202/1024017ar

Aller au sommaire du numéro

Éditeur(s)

Institut de l'économie sociale (IES)

ISSN

1626-1682 (imprimé)

2261-2599 (numérique)

Découvrir la revue

Citer cet article

Draperi, J.-F. (2001). L'économie sociale et solidaire face aux nouvelles attentes de la société. Revue internationale de l'économie sociale, (281), 5-14. https://doi.org/10.7202/1024017ar
Résumé de l'article

L'économie sociale et solidaire - nous utilisons ici cette notion vague par facilité - est à la croisée des chemins : la révolution technologique et socio-économique provoque des changements profonds concernant le rapport à l'espace et au temps, la redéfinition de la qualité des produits, les évolutions du travail salarié, le déplacement de l'action politique... Ces bouleversements rendent nécessaires l'anticipation sur les risques à venir et la construction de nouvelles formes de régulation. Face à ces risques, deux valeurs clés modèlent les nouvelles attentes : efficacité et solidarité, valeurs qui rejoignent celles de l'économie sociale et solidaire. Si celle-ci n'ignore pas ces attentes, elle ne paraît pourtant pas être aujourd'hui en mesure de les satisfaire. L'unité lui fait défaut. Il faut, sans crainte, acter les faux débats de légitimité qui la divisent, mais également faire l'effort nécessaire à leur dépassement. 


\section{L'ÉCONOMIE SOCIALE ET SOLIDAIRE FACE AUX NOUVELLES ATTENTES DE LA SOCIÉTÉ}

par Jean-François Draperi

L'économie sociale et solidaire - nous utilisons ici cette notion vague par facilité - est à la croisée des chemins : la révolution technologique et socioéconomique provoque des changements profonds concernant le rapport à l'espace et au temps, la redéfinition de la qualité des produits, les évolutions du travail salarié, le déplacement de l'action politique... Ces bouleversements rendent nécessaires l'anticipation sur les risques à venir et la construction de nouvelles formes de régulation. Face à ces risques, deux valeurs clés modèlent les nouvelles attentes : efficacité et solidarité, valeurs qui rejoignent celles de l'économie sociale et solidaire. Si celle-ci n'ignore pas ces attentes, elle ne paraît pourtant pas être aujourd'bui en mesure de les satisfaire. L'unité lui fait défaut. Il faut, sans crainte, acter les faux débats de légitimité qui la divisent, mais également faire l'effort nécessaire à leur dépassement.

\section{De nouvelles attentes de la société}

\section{Une nouvelle mesure de l'espace et du temps}

En construisant un espace-monde dans le sillage de l'économie-monde antérieurement bâtie sur la division internationale du travail (Wallerstein, 1980 ; Brunet, Dollfus, 1990), la nouvelle révolution réduit l'espace, que ce soit par la technologie avec les nouvelles formes de communication ou par l'économie avec la globalisation. Celle-ci ne signe cependant pas la mort des territoires. En effet, alors même que le marché et la consommation se mondialisent, la production s'inscrit de façon croissante dans des perspectives de développement local ou dans des logiques territoriales. Par le respect des régulations locales, par l'investissement humain auquel elle consent, par l'inaliénabilité de son capital, l'entreprise d'économie sociale et solidaire s'inscrit de façon profonde et durable dans l'espace local (Lulek, 1998). Hier avec les sociétés de secours mutuels ou les coopératives viticoles, aujourd'hui avec l'épargne de proximité ou les associations à finalité sociale et culturelle, l'économie sociale et solidaire multiplie le champ de ses activités. Précurseurs d'un renouveau de la coopération de consommation, des groupements de consommateurs informels se constituent pour procéder à des achats groupés, des associations de producteurs et de 
consommateurs émergent, et ces circuits courts à la fois augmentent la garantie des produits en matière de qualité, permettent une meilleure connaissance pour le consommateur des conditions de production de la marchandise et contribuent au maintien et au développement des emplois locaux. Lorsque les démarches s'inscrivent dans un projet de développement local, intègrent un raisonnement de filière, mettent en œuvre un partenariat intercoopératif..., le poids dans l'économie et la vie locales peut devenir déterminant, comme en témoignent l'exemple d'Ardelaine, présenté ci-après par Béatrice Barras, ainsi que l'expérience de l'agence locale pour le développement de l'économie sociale et solidaire (Aldess) introduite par Agostino Burrini.

La révolution définit une nouvelle temporalité : le temps s'accélère, l'économie est de court terme, prête à l'emploi, et à jeter. La valorisation maximale et rapide du capital domine l'esprit du néocapitalisme. Celui-ci, dirigé non plus par les managers mais par les actionnaires, traduit le pouvoir croissant des investisseurs institutionnels dans les grandes entreprises. Sa stratégie procède du triptyque fusion-acquisition-externalisation (Jacquet, 2001). Face à cette accélération avance l'idée d'un développement durable qui permet de "répondre aux besoins du présent sans compromettre la capacité des générations futures de répondre aux leurs" (Marchesi, in Capul, 1998). En 1992, la conférence de Rio de Janeiro marquait une nouvelle étape dans l'engagement en faveur du développement durable ; elle constitue aujourd'hui encore la référence pour nombre de directives. A Kyoto (1997) et à La Haye (2000), on a souligné l'urgence de la prise en compte de la dimension environnementale dans le développement durable. Mais une telle démarche suppose l'adoption d'indicateurs économiques nouveaux. Les Nations unies ont d'ores et déjà identifié cent trente-quatre indicateurs transversaux qui montrent que l'approche de l'environnement passe par l'établissement d'une évaluation qualitative à côté des mesures quantitatives (Dron, 2001).

Grâce à leur ancrage territorial, les coopératives devraient être les moyens privilégiés du développement durable. Localement, les réflexions sont avancées. Les coopératives agricoles définissent une méthodologie de bilan sociétal dont nous présentons un aspect dans ce numéro, sous la signature du président de la Confédération française de la coopération agricole, Philippe Mangin.

\section{Deux approches de la qualité}

Une dimension du développement durable réside dans les normes de définition de la qualité : les fusions et les externalisations ne sont possibles qu'à la condition de pouvoir produire partout des marchandises de qualité égale, ce qui suppose l'établissement de processus de production identiques, une main-d'œuvre sensiblement équivalente, son seul coût devenant le critère de choix. C'est l'un des enjeux de l'International Standard Organization (ISO) [Segrestin, 1996]. A l'inverse de la démarche de définition des normes ISO, l'établissement des normes patrimoniales, qui consiste en une « explicitation de pratiques " respectant les usages loyaux et constants et le maintien de la qualité (Sainte-Marie, Prost, Casabianca, Casalta, 1995), 
fait des spécificités locales, naturelles et culturelles, les critères incontournables de la qualité. La définition de qualités patrimoniales inspire de nombreuses organisations professionnelles et interprofessionnelles qui contractualisent leur démarche dans le cadre de labels et d'appellations de tous ordres. Ce processus n'est pas remis en cause par la globalisation : le nombre de produits en appellation d'origine contrôlée (AOC) en France est passé d'une centaine à quatre cents au cours des vingt dernières années. Cette relocalisation ne concerne pas seulement les activités patrimoniales. Les produits nécessitant de la haute technologie se concentrent aussi dans des bassins d'emploi très spécifiques, en raison d'une qualité particulière de la main-d'œuvre et d'un environnement particulier (Benko, Lipietz, 1995). La qualité que l'on a appelée patrimoniale est donc plus largement une qualité de spécificité qui tantôt s'articule avec, tantôt joue contre la normalisation de type ISO au sein même du processus de globalisation de l'économie. Bien entendu, cette approche qualitative ne concerne pas que l'économie sociale et solidaire, loin s'en faut. Elle s'inscrit cependant dans une démarche proche : en effet, elle ne postule pas de la dissolution des solidarités et des valeurs traditionnelles, mais au contraire les renouvelle dans le contexte de la société informationnelle. Cette approche nécessite également l'établissement de formes locales de régulation sociale et économique (Sainte-Marie, Casabianca, 1995), qui sont généralement initiées par des associations professionnelles coopérant dans des organisations d'économie mixtes (comme les comités interprofessionnels). Ce sont, en règle générale, les pratiques coopératives et contractuelles qui président à leur fonctionnement. Les organisations coopératives de producteurs sont d'ailleurs souvent des acteurs majeurs de ce type de régulation (Draperi, 2001).

L'évolution des marchés indique que les consommateurs se tournent de plus en plus massivement vers des produits de qualité patrimoniale, en particulier dans l'alimentation, secteur dans lequel la sensibilité au risque est particulièrement forte. La prévention des risques (environnementaux, alimentaires, sanitaires...) joue un rôle déterminant sur la consommation. N'est-ce pas la mission même de la mutualité de prévoyance et de nombreuses associations que de répondre à ces nouveaux besoins, comme elles l'ont fait hier pour l'éradication des maladies, la tuberculose et la poliomyélite, en matière de prévention - de l'alcoolisme, de la drogue, puis du sida -, dans l'établissement de services aux personnes âgées ou handicapées?

\section{Le travail qui épuise... ou exclut lorsqu'il manque}

L'économie libérale est coûteuse, en termes humains et environnementaux. La flexibilité et l'intensification du travail font des dégâts sur chacun : angoisse, stress, usure mentale pour les cadres ; insatisfaction, maladies musculo-squelettiques pour les activités manuelles taylorisées ; fragilisation, chômage de masse, exclusion, effondrement de l'économie locale, désertification, gâchis pour la collectivité, lorsque le travail est absent. Comme le résume Daniel Cohen, "ce ne sont plus les machines qui tombent en panne, ce sont les hommes eux-mêmes» (Cohen, 1999). 
L'intensification du travail est un changement majeur lié à l'actuelle transformation du modèle productif. Le travail posté augmente, les délais fixés à l'accomplissement des tâches diminuent : $60 \%$ des salariés de l'industrie française sont soumis à des normes de production ou à des délais à respecter en une journée maximum; dans le bâtiment, ce chiffre est plus important encore. Dans le secteur tertiaire, entre 1984 et 1998, le chiffre des salariés soumis à des telles normes est passé de 19 à $43 \%$. L’essor du néotaylorisme s'exprime encore par des horaires décalés plus fréquents (Charpin, 2000)

Face à l'intensification du travail entrânant la détérioration de la santé, et face à l'installation dans la précarité et l'exclusion de certaines populations fragiles, les attentes sociales s'expriment à travers la prévention des risques liés aux maladies professionnelles et la revendication du droit au travail. Celui-ci apparait avec la famille comme l'une des valeurs centrales de notre société. Le travail reste le vecteur central de l'insertion. "C'est le moyen d'assurer la vie matérielle et structurer le temps et l'espace, c'est le lieu de l'expression de la dignité de soi et des échanges sociaux... C'est dans la mesure où lindividu est autonome grâce à son travail quill peut être en même temps autonome dans sa pratique de citoyen" (Schnapper, 2000).

La question de l'emploi, du travail, d'un travail qualifié, constitue sans doute le défi majeur d'une autre économie. C'est la finalité même du mouvement coopératif qui est ici posée : l'éducation, le développement de la personne est le premier but de l'association et de la coopération, et comme le disait Fauquet, "si la coopérative était défaillante dans sa tâche morale, elle serait défaillante dans sa tâche économique "(Fauquet, 1935). De ce point de vue, au cours de son histoire, l'économie sociale et solidaire s'est donné au moins trois missions : 1) définir un autre rapport au travail ; 2) soutenir en leur proposant du travail les personnes en difficulté d'insertion (lire dans ce numéro la présentation de l'association Les Ateliers de la Bergerette) ; 3) déceler les potentialités et créer des emplois. C'est sans aucun doute le premier enjeu qui apparait aujourd'hui le plus lointain. On se souvient que les internationalistes de 1864 s'étaient fixé comme objectif de libérer le travail du salariat au moyen de la multiplication d'associations coopératives (Kriegel, 1964). L’ambition d'abolir le salariat était sans doute " le seul trait d'union authentique " entre le socialisme utopique de Charles Fourier et le socialisme marxiste (Tacussel, 2000). Non seulement le mouvement ouvrier s'est très vite détourné de ce but, mais le salariat continue de progresser (Montbrial, Jacquet, 2000 ; Lallement, 2000). C'est dans le cadre du travail informel et dans les régions les plus paupérisées du globe que le « travail associé » est le plus présent. La coopération elle-même a évolué, au moins partiellement, indépendamment du sociétariat ouvrier - "révision déchirante ", dira Charles Gide -, en admettant de salarier des ouvriers non coopérateurs. Ce problème est aujourd'hui réactualisé. Par exemple, on observe depuis peu des évolutions rapides dans la formation des salariés, des dirigeants et des administrateurs : la demande s'amplifie, les formations se renforcent, les études et les recherches se multiplient, témoignant d'une nouvelle 
approche du travail et de son organisation au sein de nombreuses entreprises de l'économie sociale (Revue internationale de l'économie sociale-Recma, 2001). Or, toute formation " coopérative " vers des membres, bénévoles ou salariés, interroge l'identité associative, coopérative, mutualiste de l'entreprise. La création d'emplois, la solidarité avec les sans-emploi, le rapport au travail sont des problèmes qui se posent évidemment de façon différente selon les types d'entreprises et les secteurs d'activité de l'économie sociale et solidaire, mais la même question se pose néanmoins à toutes les organisations, dès l'élaboration de leur projet initial jusqu'au mode de répartition et à la destination des excédents de gestion : s'il existe une politique de management humain spécifique à l'économie sociale et solidaire, c'est à partir d'indicateurs de ce type - une attention originale à l'emploi, la solidarité, l'organisation du travail, la formation des membres... - qu'elle peut être identifiée.

Ce questionnement sur l'évolution du travail est d'autant plus important que l'intensification du travail a accompagné à la fois un accroissement des inégalités entre les niveaux de vie et, pour la majorité des pays de l'hémisphère nord, une augmentation du produit intérieur brut (PIB).

La pauvreté proprement dite provient aujourd'hui massivement de l'exclusion du processus de production, alors que jusqu'il y a une trentaine d'années elle était essentiellement issue de la surexploitation du travail. D'où l'essor formidable du thème de la solidarité et de l'économie solidaire. La solidarité peut cependant n'être qu'un pis-aller, si elle est disjointe de l'inégalité qui l'oblige : on s'accorde à considérer qu'il vaut mieux une société de travail qu'une société d'assistance, et la mutualisation volontaire que la solidarité contrainte.

Cette "désafiliation ", selon le terme de Robert Castel, provoque une nouvelle attente de lien social, dont témoigne l'essor associatif. Cet essor montre qu'alors que s'étend la mondialisation économique, les liens interpersonnels, les échanges de services, de savoirs, de produits échappant à la sphère marchande, loin de se réduire, se multiplient et s'enrichissent : l'entraide, le sport, la culture, l'activité sociale suscitent la créativité associative. On s'associe pour être ensemble ou pour faire ensemble, pour faire ce qui plaît avec ceux que l'on choisit. Selon le langage de Fourier, c'est la recherche du "charme sériaire » de la "composite ", qui associe le plaisir de faire à celui du choix des compagnons (Fourier, 1966) : au total, pour le cas français, quelque chose comme dix millions de bénévoles avec une moyenne de cinq heures par semaine (Archambault, Boumendil, 1997).

\section{Démocratie politique et démocratie civile}

Comme l'a récemment montré Pierre Rosanvallon, "la démocratie représentative s'est imposée dans son principe au moment où elle s'est fragilisée dans son fonctionnement " (Rosanvallon, 2000). Notre période connaît simultanément le plus grand nombre de gouvernements démocratiquement élus et une désaffection des urnes. Ce que l'auteur appelle "le déclin de la volonté" n'est-il pas un transfert de la volonté de changement du champ politique 
des partis vers le champ social de la société civile ? Tout se passe comme si l'enjeu démocratique qui visait hier à établir un droit de participation se déplaçait pour établir aujourd'hui un droit de l'individu. L'implication associative définirait un nouvel essor de la démocratie, mais d'une démocratie civile à la fois différente et complémentaire de la démocratie politique. C'est ce que l'Etat reconnait aujourd'hui, sur une base proche de ce que François Bloch Lainé définissait il y a quelques années comme les «spécificités méritoires " des associations : fonction d'avant-garde et d'innovation sociale, capacité à mobiliser des ressources gratuites, accompagnement social et personnalisé des bénéficiaires des interventions et aptitude à renforcer les liens sociaux par l'exercice de la démocratie au quotidien (Bloch

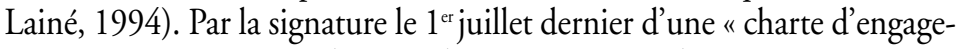
ments réciproques entre l'Etat et les associations ", l'Etat se met en position de " donner et recevoir » vis-à-vis du secteur associatif : la charte précise en effet que l'Etat " reconnaît l'importance de la contribution associative à l'intérêt général dont il est le garant" (Charte d'engagements réciproques entre l'Etat et les associations, 2001). Nous reviendrons sur cette question dans le numéro 282 d'octobre 2001, qui sera consacré aux associations. Le travail en matière de formation initié par la Délégation interministérielle à l'innovation et à l'économie sociale, sous l'impulsion de Hugues Sibille, et présenté dans ce numéro par Scarlett Courvoisier témoigne de ce changement historique qui touche les relations entre l'Etat et les associations.

\section{Solidarité et efficacité : réconcilier les valeurs de la démocratie et de l'économie}

Les attentes des Français dans la vie économique et sociale se façonnent autour de deux valeurs clés qui sont la solidarité et l'efficacité. Solidarité avec les personnes en difficulté par l'essor d'une nouvelle action sociale et d'une régulation sociale de l'Etat, solidarité avec les jeunes générations par l'attachement à la famille organisée autour de l'enfant, solidarité avec les générations futures par l'organisation d'une économie durable qui préserve l'avenir. Efficacité par et pour le maintien de la croissance et du pouvoir d'achat, pour permettre l'investissement, l'épargne et l'emploi. Et solidarité et efficacité ensemble par souci de justice, de conciliation entre des valeurs universelles des droits de l'homme et une économie source d'inégalités. C'est ce dont témoigne l'étude de François Mayaux, publiée dans la seconde partie de ce numéro, sur la mobilité professionnelle des cadres de l'économie marchande vers l'économie sociale.

Les valeurs fondatrices de l'économie sociale et solidaire rejoignent celles qui modèlent les nouvelles attentes sociales : volontariat, responsabilité, éducation, égalité, solidarité. Mais les règles de l'économie sociale s'inscrivent, de fait, dans l'économie, et l'entreprise coopérative ne vit pas pour respecter des principes, mais pour servir des besoins. Or, il y a souvent tension entre les valeurs et les règles parce que la vie économique est dominée par un secteur capitaliste qui n'obéit pas aux règles démocratiques. La tension vécue par les coopératives est inhérente au projet de construire une 
économie humaine. Cette tension est au fondement des innovations de l'économie sociale, comme le montre la contribution d'Yves Vaillancourt et Louis Favreau.

\section{Pour une approche de l'économie sociale et solidaire}

Voilà une trentaine d'années que le concept d'économie sociale a été réactualisé. La récente rencontre de Gävle en Suède, sur laquelle le secrétaire d'Etat à l'Economie solidaire, Guy Hascoët, a accepté de s'exprimer ci-après, a permis de mesurer le chemin parcouru : à la fois modeste et réel, ainsi qu'en témoigne la contribution de Hans Münkner dans ce numéro, qui éclaire l'évolution théorique du concept d'économie sociale en Allemagne. La "social economy " émerge à l'échelon européen, elle apparaît dans le langage politique aussi bien au niveau national qu'au niveau local (Westlund, 2001).

Comme chacun des contributeurs de ce numéro le constate, l'économie sociale et solidaire n'est cependant pas encore définie de façon réellement claire et univoque. La définition de l'économie sociale et solidaire pose de nombreux problèmes, particulièrement en France. En général, on définit l'économie sociale à partir de statuts juridiques, et sa mesure s'appuie largement sur la quantification, par exemple celle des membres impliqués dans les entreprises concernées et l'activité économique réalisée (Jeantet, 1999). L'économie alternative se définit essentiellement à partir d'initiatives caractérisées par leur projet, fréquemment identifié en termes d'autonomie et de solidarité (Archimbaud, 1995). L'économie solidaire est approchée dans les termes d'un projet qui associe la réciprocité au marchand et à la redistribution (Laville, 1994). Cette multiplicité des approches constitue certes une richesse, mais elle ne doit pas nuire à la réaffirmation d'une unité dont l'économie sociale et solidaire a besoin.

Malheureusement, le débat récemment ouvert entre économie solidaire et économie sociale aborde fréquemment les faits relatifs aux valeurs de façon biaisée. En bref, deux discours antagoniques prennent forme. D'un côté, on croit comprendre que l'économie sociale servirait l'intérêt des sociétaires sans se préoccuper de l'intérêt général, alors que l'économie solidaire serait une économie de la réciprocité qui servirait l'intérêt général. Parallèlement, l'intérêt égoïste des premiers relèverait de la logique économique, alors que l'intérêt général poursuivi par les seconds s'accorderait avec l'altruisme. De l'autre côté, on émet que l'économie sociale est un mouvement d'entreprises bien identifiables, ayant des statuts propres, une activité économique et sociale certaine, alors que l'économie solidaire constituerait un discours dont on ne verrait pas bien la traduction pratique sinon à travers les politiques sociales de l'Etat. Ces interprétations, largement répandues l'une et l'autre, ne valent que si l'on admet deux définitions qui simplifient exagérément chacune des démarches et qui, de surcroît, ne peuvent pas être opposées. 
- On ne peut pas affirmer que l'économie sociale procède de la logique de l'intérêt alors que la solidarité entre membres est au fondement même de toute organisation coopérative, mutualiste et associative. Que la logique de l'intérêt soit présente est une évidence : peut-on dire où elle n'est pas ? Il faut plutôt retourner l'interrogation : "La véritable question est de savoir sìl existe une pluralité de motivations ou si l'intérêt personnel est le seul motif qui guide les êtres humains ", affirme Amartya Sen (Sen, 1993), et la question concrète est plutôt : avec qui, et jusqu'où, est-on solidaire? C'est ce que montre Franck Giraud dans ce numéro, à travers l'analyse de la gestion de la relation adhérent dans la mutualité.

On ne peut pas non plus réduire l'économie solidaire à une politique publique alors que les acteurs associatifs et coopératifs qui agissent - professionnellement ou non - à des fins de solidarité revendiquent un engagement personnel sans lequel la volonté publique ne pourrait s'exprimer. Au-delà de la fonction qu'elle remplit, l'économie solidaire réunit bien des acteurs sociaux poursuivant un projet commun. (Ceci est aussi vrai de l'économie alternative, même si son existence est beaucoup moins reconnue que celle de l'économie solidaire.)

- Ces deux perspectives ne peuvent opposées lorsqu'on les simplifie en un ensemble de pratiques (d'économie sociale), d'une part, et un ensemble de propositions projectives (d'économie solidaire), de l'autre. Comme l'a constaté J.-B.-A. Godin, le phalanstère de Fourier est une utopie écrite, alors que son familistère est une entreprise : il est plus facile de magnifier l'utopie phalanstérienne que le familistère de Guise et d'affirmer que le phalanstère est plus vertueux que le familistère. Inversement, il est plus aisé de mesurer la réalité du familistère que celle du phalanstère et de conclure que seul le familistère existe réellement. On voit bien les limites d'une telle argumentation : on ne peut pas mesurer terme à terme l'idéel et le matériel (Desroche, 1976). Il y a un "hiatus insurmontable " entre le concept et le réel, comme le disait Max Weber. Il faut pourtant bien avancer. Ainsi qu'en témoigne la démarche initiée à l'occasion de la définition de la société coopérative d'intérêt collectif (SCIC), dont Lucile Manoury offre une remarquable analyse, ce qui est en jeu est la mise en œuvre de pratiques collectives en lien avec des principes et des valeurs. C'est également l'une des leçons de la recherche de Michel Garrabé, Laurent Bastide et Catherine Fas, dans le numéro 280, à partir de la notion d'utilité sociétale (Garrabé, Bastide, Fas, 2001) qui leur permet de présenter ci-dessous une évaluation du secteur de l'économie sociale en Languedoc-Rousillon. C'est aussi dans cette voie qu'avance Marie-Claire Malo dans sa contribution à ce numéro sur la gestion stratégique de la coopérative et de l'association d'économie sociale.

Lobjet de l'économie sociale et solidaire serait ainsi de définir des règles organisationnelles en relation avec un projet dont les valeurs fondamentales s'organisent autour du respect du droit de la personne et de la subordination de l'économie à ce droit : règles juridiques, mais aussi règles informelles construites dans la vie quotidienne de l'organisation. 
Le premier postulat de cette proposition est que toute action a un fondement normatif qui permet de la justifier. Mais le second est que l'on ne peut opposer terme à terme un fondement altruiste et un fondement égoïste de l'action : "La solidarité est inséparable du calcul " (Reynaud, 1993). Elle en est inséparable parce que l'action collective ne se fonde pas uniquement sur le partage d'intérêts communs, mais également sur l'existence de contraintes de réciprocité. Parce que normative, l'interaction définit toujours une offre d'engagement et de réciprocité. C'est finalement l'action collective elle-même qui constitue les acteurs et c'est leur façon d'échanger, de négocier, de coopérer qui définit les règles. La coopération définit ainsi moins un système de règles établies - construit juridique nécessaire mais non suffisant - qu'un processus particulier de construction de règles : à l'origine du projet, comme à la genèse des règles, il existe une régulation - c'est-à-dire un processus de production des règles spécifique qui caractérise l'entreprise d'économie sociale et solidaire, quelque chose comme un groupe fonctionnant de façon démocratique. Cette régulation procède d'une relation originale entre les acteurs individuels et le collectif. De ce point de vue, le mouvement qui porte l'entreprise d'économie sociale et solidaire est en premier lieu un mouvement éducatif. La participation des associés à la construction de nouvelles règles définit de nouveaux savoirs issus de l'action, expérienciels : leur apprentissage collectif constitue le premier acquis de l'économie sociale et solidaire. Sous cet angle, le rapprochement entre C. Fourier et J.-B.-A. Godin est fertile. En cherchant à promouvoir simultanément l'éducation des hommes et l'innovation dans l'entreprise - l'une générant l'autre -, et à l'opposé de doctrines empreintes d'idéalisme ou de mysticisme qui s'appuient sur un autre hominis et rêvent d'une nouvelle humanité, leur action vise à donner aux hommes tels qu'ils sont les moyens de mieux vivre ensemble. 


\section{Bibliographie}

Archambault, E., Boumendil, J., "Dons et bénévolat en France ", Revue internationale de l'économie sociale-Recma, n $267,1997$.

Archimbaud, A., «L'économie alternative, forme radicale de l'économie sociale", Revue internationale de l'économie sociale-Recma, $\mathrm{n}^{\circ} 256,1995$.

Benko, G., Lipietz, A., "De la régulation des espaces aux espaces de régulation ", in Boyer, R., Saillard, Y., La Découverte, 1995, p. 293.

Bloch Lainé, F., "Les spécificités méritoires des associations de service social ", Revue internationale de l'économie sociale-Recma, $\mathrm{n}^{\circ} 251,1994$, et $\mathrm{n}^{\circ}$ 275-276, 2000.

Brunet, R, Dollfus, O., Mondes nouveaux, Belin-Reclus, 1990. Bureau international du travail, Promotion des coopératives, 2000.

Capul (J.-Y.), " Les indicateurs économiques en question ", Cahiers français, $\mathrm{n}^{\circ}$ 286, La Documentation française, mai-juin 1998.

Castel, R., Les métamorphoses de la question sociale, Fayard, 1995.

Charpin (J.-M.), Commissariat général du plan, Rapport sur les perspectives de la France, rapport au Premier ministre, La Documentation française, 2000.

Charte d'engagements réciproques entre l'Etat et les associations, $1^{\text {er }}$ juillet 2001, "Préambule ".

Cohen, D., Nos temps modernes, Flammarion, 1999, p. 61

Defourny, J., Develtere, B., Fonteneau B., L'économie sociale au Nord et au Sud, 1999.

Delabre, G., Gautier, J. M., Godin et le familistère de Guise, textes choisis et Vervins, Centre de documentation de la Thiérache, 1983.

Desroche, H., La société festive, Seuil, 1975.

Develtere, P., Economie sociale et développement, De Boeck, 1998.

Données sociales, La Documentation française, 2000.

Draperi, J.-F., "Coopération et régulation des marchés ", Cahiers du Griot, n², Cnam, 2001.

Dron, D., "Environnement : les enjeux du prochain siècle ", in Ramses, Les grandes tendances du monde, Dunod, 2001, p. 95.

Durkheim, E., De la division du travail social, Puf, 1930. Fauquet, G., Le secteur coopératif, 1935, édition de 1965 , AISC, p. 44.

Fourier, C., Le nouveau monde industriel et sociétaire, 1966 (la première édition est de 1829).

Garrabé, M., Bastide, L., Fas, C., "Identité de l'écono- mie sociale et de l'économie solidaire ", Revue internationale de l'économie sociale-Recma, $n^{\circ}$ 280, 2001.

Jacquet, P., " Nouvelle économie : du virtuel au réel ", in Ramses, Les grandes tendances du monde, Dunod, 2001, p. 37. Jeantet, T., L'économie sociale européenne, Ciem, 1999.

Kriegel, A., Les internationales ouvrières, Puf, 1964.

Lallement, M., "Globalisation : où en sommes-nous?", Cahiers du Griot, n 1, Cnam, 2000.

Laville, J.-L. (dir.), L'économie solidaire, une perspective internationale, Desclée de Brouwer, 2000.

Lipietz, A., Pour le tiers secteur, La DécouverteLa Documentation française, 2001.

Luleck, M., Ambiance Bois, Utovie, 1998.

Montbrial (T. de), Jacquet (P.), Les grandes tendances $d u$ monde, Ramses, Dunod, 2000.

Parodi, M. (dir.), La question sociale en France depuis 1945, Armand Colin, 2000.

Reynaud, J.-D., Les règles du jeu, 1993, “Préface », p. III.

Rosanvallon, P., La démocratie inachevée, Gallimard, 2000.

Sen, A., Ethique et économie, Puf, 1993, p. 22.

Sainte-Marie, C. de, Prost, J.-A., Casabianca F., Casalta, E., "La construction sociale de la qualité ", in Nicolas, F., Valceschini, E. (dir.), Agro-alimentaire : une économie de la qualité, Inra Economica, 1995, p. 185.

Sainte-Marie, C. de, Casabianca F., "Innover dans les productions patrimoniales ", Cahiers d'économie et de sociologie rurale, $\mathrm{n}^{\circ} 37,1995$, p. 171 et suiv.

Segrestin, D., " La normalisation de la qualité et l'évolution de la relation de production ", Revue d'économie industrielle, $\mathrm{n}^{\circ} 75,1996$, p. 305.

Schnapper, D., cité par Charpin, J.-M., Commissariat général du plan, Rapport sur les perspectives de la France, rapport au Premier ministre, La Documentation française, 2000, p. 65.

Tacussel, P., Charles Fourier, le jeu des passions, Desclée de Brouwer, 2000, p. 208.

Wallerstein, I., Le système du monde du XV eiècle à nos jours, Flammarion, 1980.

Weber, M., Essai sur la théorie de la science, Plon, 1955.

Westlund, H., Social Economy in Sweden, Svenska Institutet för social ekonomi, 2001

Economie et solidarités, "La gouvernance locale ", Presses de l'université du Québec, vol. 30, n² 2, 1999.

Revue internationale de l'économie sociale-Recma, "Un siècle d'économie sociale ", $\mathrm{n}^{\circ}$ 275-276, 2000.

Revue internationale de l'économie sociale-Recma, $\mathrm{n}^{\circ} 280$, "La formation en économie sociale dans tous ses états ", 2001, p. 8 . 